PSS PROCEEDINGS

\title{
Chiral dynamics of few-nucleon systems: recent developments
}

\section{Evgeny Epelbaum*}

Institut für Kernphysik (IKP-3) and Jülich Center for Hadron Physics, D-52425 Jülich, Germany and

Helmholtz-Institut für Strahlen- und Kernphysik (Theorie) and Bethe Center for Theoretical Physics, Universität Bonn, D-53115 Bonn, Germany

E-mail: e.epelbaumefz-juelich.de

I overview the current status of chiral effective field theory in the few-nucleon sector and discuss selected recent applications.

8th Conference Quark Confinement and the Hadron Spectrum September 1-6 2008

Mainz, Germany

${ }^{*}$ Speaker. 


\section{Introduction}

A quantitative description of the nuclear force is one of the cental topics in nuclear physics which remains to be open despite the long history and extensive research. At the fundamental level of quantum chromodynamics (QCD), the nuclear force can be viewed as a residual color interaction between colorless hadrons similar to intermolecular van der Waals-forces. Unfortunately, the nonperturbative nature of QCD in the low-energy region relevant for nuclear physics does not allow, at least at present, to directly derive the nuclear force, see however Refs. [1] for some exciting developments along this line within lattice QCD. Alternatively, nuclear forces can be derived using chiral effective field theory (EFT) [2], which allows to explore the consequences of the spontaneously broken approximate chiral symmetry of QCD in a systematic and model independent way, see Refs. [3, 4, 5] for recent review articles on this subject. In this talk I overview some recent developments and consider selected applications in this field. The manuscript is organized as follows. In section 2 I discuss the current status of nuclear forces based on chiral EFT and applications to few-baryon systems, the reaction $\gamma d \rightarrow \pi^{+} n n$ as a tool to extract the neutron-neutron scattering length and recent attempts towards inclusion of the $\Delta$-isobar as an explicit degree of freedom. A short summary and outlook are given in section 3 .

\section{Selected topics in chiral EFT for few-nucleon systems}

Most of the few-nucleon studies based on chiral EFT utilize the formulation involving only pions and nucleons as explicit degrees of freedom and make use of the heavy-baryon expansion. In this framework, the expressions for the nuclear force have been worked out up to next-tonext-to-next-to-leading order $\left(\mathrm{N}^{3} \mathrm{LO}\right)$ in the chiral expansion with the only exception of certain three-nucleon force contributions which are currently been worked out. The chiral power counting supports the observed hierarchy of nuclear forces with many-body interactions yielding only minor corrections to the two-nucleon force ( $2 \mathrm{NF})$. At $\mathrm{N}^{3} \mathrm{LO}$, the $2 \mathrm{NF}$ involves the one- two- and three-pion exchange contributions as well as 24 isospin-invariant short-range contact operators. All low-energy constants (LECs) entering the multiple-pion exchange contributions are known from the pion-nucleon system [6]. The LECs accompanying the contact interactions have been determined from a fit to low-energy two-nucleon data utilizing the cutoffs in the Lippmann-Schwinger equation in the range $\Lambda=450 \ldots 600 \mathrm{MeV}[7,8]$. Both available $\mathrm{N}^{3} \mathrm{LO}$ analyses of [7] and [8] yield accurate results for the neutron-proton phase shifts up to $E_{\text {lab }} \sim 200 \mathrm{MeV}$ and the deuteron observables and also agree with each other within the theoretical uncertainty. A much bigger cutoff variation with $\Lambda<4 \mathrm{GeV}$ was considered in [9] using the leading-order approximation for the long-range interaction (i.e. one-pion exchange potential) and employing a different power counting for contact interactions, see however the discussion in Refs. [10, 11]. Yet different approach based on the short-range boundary conditions is followed by the Granada group, see [12] for a recent overview. More work is needed in the future in order to clarify the relation between the well-established chiral expansion of the nuclear potential and the scattering amplitude.

Theoretical studies of three- and more-nucleon systems have been carried out up to next-tonext-to-leading order $\left(\mathrm{N}^{2} \mathrm{LO}\right)$. At this order, one has to take into account the first three-nucleon force contributions $[13,14]$ which depend on two new LECs. These LECs can be determined by 

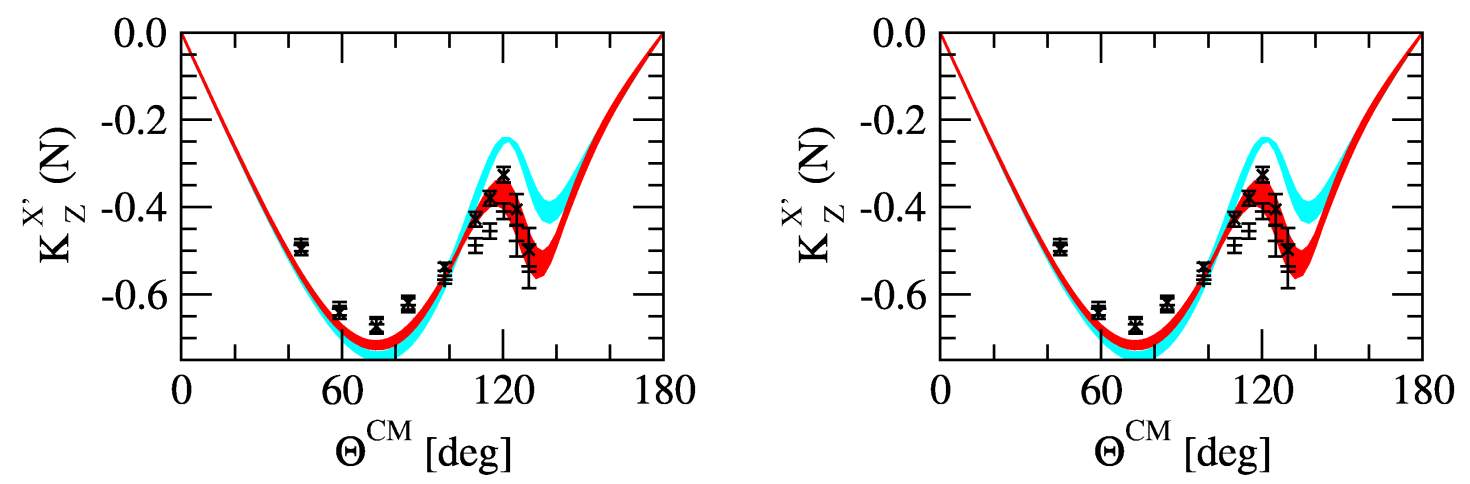

Figure 1: The proton-to-proton (left) and proton-to-deuteron (right) polarization transfer coefficients in $d(\vec{p}, \vec{p}) d$ and $d(\vec{p}, \vec{d}) p$ reactions at $E_{p}^{\mathrm{lab}}=22.7$ [17]. Light (dark) shaded bands depict the results at NLO $\left(\mathrm{N}^{2} \mathrm{LO}\right)$.

e.g. fitting the ${ }^{3} \mathrm{H}$ binding energy and either the $n d$ doublet scattering length [14], the ${ }^{4} \mathrm{He}$ binding energy [15] or the properties of light nuclei [19]. Most of the three-nucleon scattering observables are reasonably well described at $\mathrm{N}^{2} \mathrm{LO}$ [14], see Fig. 1 for a representative example, with few exceptions such e.g. as the differential cross section in the so-called symmetric constant relativeenergy neutron-deuteron breakup configuration [18]. The theoretcal uncertainty becomes sizeable at intermediate and higher energies which is consistent with the results in the two-nucleon systems and calls for a complete $\mathrm{N}^{3} \mathrm{LO}$ analysis. The spectra of light nuclei have also been considered using chiral EFT. For example, one finds for the $\alpha$-partical binding binding energy $B=24.4 \ldots 28.8 \mathrm{MeV}$ $(B=27.8 \ldots 29.6 \mathrm{MeV})$ at $\mathrm{NLO}\left(\mathrm{N}^{2} \mathrm{LO}\right)$ [4] to be compared with the experimental value $B_{\exp }=$ 28.3 MeV. Various properties of certain S-shell and P-shell nuclei with $A \leq 13$ have been studied within the no-core shell model [19]. One observes that the inclusion of the chiral three-nucleon force leads to an improved description of the data.

The first applications of chiral EFT in the Weinberg power counting scheme to few-baryon systems with strangeness are also available. In particular, baryon-baryon interactions with strangeness $S=-1,-2$ have been studied at leading order in Refs. [20, 21] where a good description of the 35 low-energy hyperon-nucleon scattering data was obtained. An extension of these studies to next-to-leading order is in progress.

Chiral effective field theory was also successfully applied to various few-nucleon reactions with electroweak and pionic probes. As a representative example, I briefly discuss here the recent application to the reaction $\gamma d \rightarrow \pi^{+} n n$ focusing on the possibility to extract the neutron-neutron scattering length $a_{n n}$. Notice that this fundamental observable is still rather poorly known due to the lack of a neutron target. In particular, recent determinations from the neutron-deuteron breakup reaction yield controversial results: $a_{n n}=-18.7 \pm 0.6 \mathrm{fm} \mathrm{[22]} \mathrm{and} a_{n n}=-16.3 \pm 0.4 \mathrm{fm} \mathrm{[23].} \mathrm{As}$ demonstrated in [24] and visualized in the left panel of Fig. 2, $a_{n n}$ can be reliably extracted from the reaction $\gamma d \rightarrow \pi^{+} n n$ using a properly chosen kinematics. Notice further that a similar proposal for the extraction of $a_{n n}$ from the reaction $\pi^{-} d \rightarrow \gamma n n$ is considered in [25].

The last topic I would like to discuss is the role of the $\Delta$-isobar for nuclear forces and lowenergy few-nucleon reactions. The chiral expansion for the nuclear force is known to exhibit a somewhat unnatural convergence pattern. For example, the subleading two-pion exchange (TPE) contribution to the $2 \mathrm{NF}$ force at $\mathrm{N}^{2} \mathrm{LO}$ turns out to be numerically much bigger than the leading 

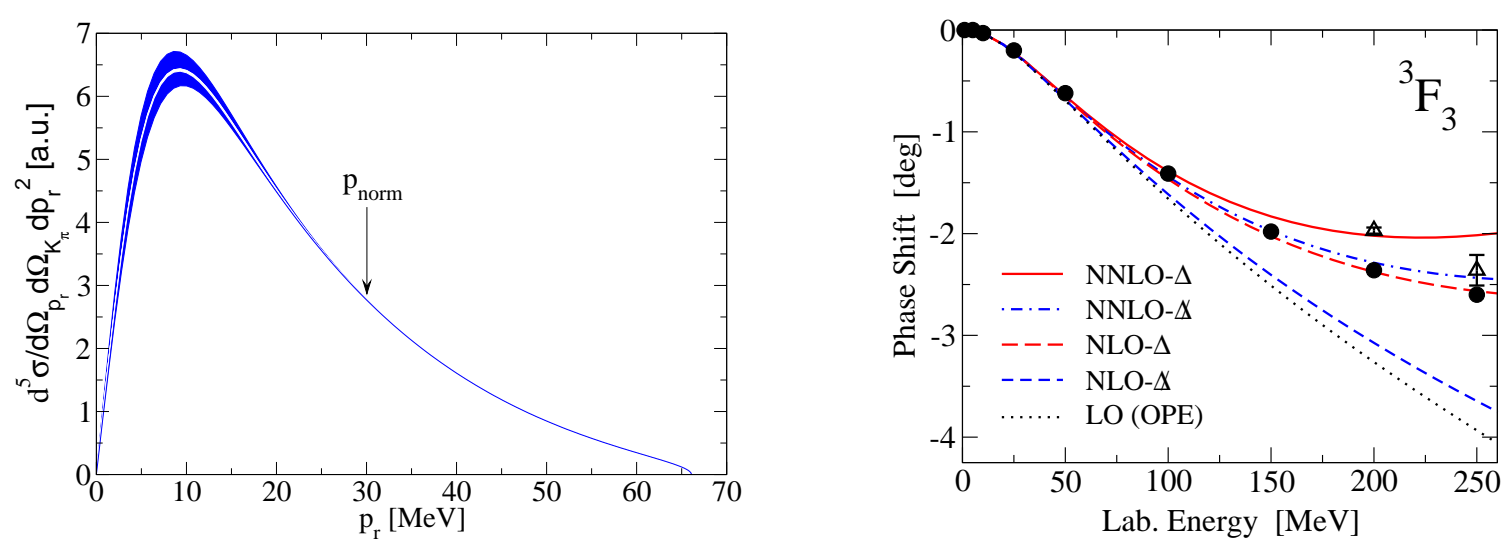

Figure 2: Left panel: differential cross section for the reaction $\gamma d \rightarrow \pi^{+} n n$ to $a_{n n}$ at the excess energy $Q=$ 4.65 $\mathrm{MeV}$ as a function of the relative momentum $p_{r}$ between the two neutrons for a polar angle of the two neutrons $\theta_{r}=90^{\circ}$. The dark-shaded band shows the sensitivity to $a_{n n}$ varied in the range $a_{n n}=-18 \ldots-20$ $\mathrm{fm}$. The light-shaded band gives the estimated theoretical uncertainty for the determination of $a_{n n}$. Right panel: ${ }^{3} \mathrm{~F}_{3} \mathrm{NN}$ scattering length with and without $\Delta$ 's calculated in the first Born approximation using the spectral-function cutoff $700 \mathrm{MeV}$ in comparison with the Nijmegen (filled circles) and Virginia Tech (open triangles) partial wave analysis.

one at NLO. A similar pattern is observed also for three-pion exchange and the charge-symmetrybreaking TPE potentials. This can be traced back to the large values of the dimension-two LECs $c_{3,4}$ which is well understood in terms of resonance saturation. In particular, the $\Delta$-isobar provides the dominant (significant) contribution to $c_{3}\left(c_{4}\right)$. Given its low excitation energy, $m_{\Delta}-m_{N}=293$ $\mathrm{MeV}$, and strong coupling to the $\pi N$ system, one can expect that the explicit inclusion of $\Delta$ in EFT utilizing the so-called small scale expansion (SSE) will allow to resum a certain class of important contributions and improve the convergence as compared to the delta-less theory. The dominant contribution of the $\Delta$-isobar to the $2 \mathrm{NF}$ in the SSE arises at NLO [26]. In [27] the corrections at $\mathrm{N}^{2} \mathrm{LO}$ have been worked out and the relevant LECs accompanying the $\pi \pi N N$ and $\pi N \Delta$ vertices were determined from $\pi N \mathrm{~S}$ - and P-wave threshold coefficients. As expected, the LECs $c_{2,3,4}$ are strongly reduced in the $\Delta$-full EFT which results in a much more natural convergence pattern for the TPE potential. Contrary to the $\Delta$-less theory, the numerically dominant contributions to the TPE potential are now generated at NLO with the $\mathrm{N}^{2} \mathrm{LO}$ terms yielding typically only modest corrections. The improved convergence is also clearly visible in peripheral NN partial waves which were calculated using the Born approximation and, at this order, are only sensitive to the OPE and TPE potentials, see the right panel of Fig. 2 for a representative example. Further recent applications along these lines are described in Refs. [28, 29].

\section{Summary and outlook}

In this talk I discussed the status of chiral EFT in the few-nucleon sector. Low-energy nucleonnucleon data are accurately described at $\mathrm{N}^{3} \mathrm{LO}$ in the chiral expansion. The results for $3 \mathrm{~N}$ scattering and the properties of light nuclei are so far only available at $\mathrm{N}^{2} \mathrm{LO}$. While most of the calculated $3 \mathrm{~N}$ observables are in a reasonable agreement with the data, the theoretical uncertainty at this order is rather large. It is, therefore, mandatory to extend this studies to $\mathrm{N}^{3} \mathrm{LO}$. I have also discussed 
the recent application of the reaction $\gamma d \rightarrow \pi^{+} n n$ which was shown to be well suited to extract the neutron-neutron scattering length. Inclusion of the $\Delta$-isobar as an explicit degree of freedom is another important topic and is expected to improve the convergence of the chiral expansion and possibly to extend its applicability range to higher energies.

I am grateful to all my collaborators and thank the organizers for making this exciting conference possible.

\section{References}

[1] S. Aoki, T. Hatsuda and N. Ishii, arXiv:0805.2462 [hep-ph].

[2] S. Weinberg, Phys. Lett. B 251, 288 (1990).

[3] P. F. Bedaque and U. van Kolck, Ann. Rev. Nucl. Part. Sci. 52 (2002) 339.

[4] E. Epelbaum, Prog. Part. Nucl. Phys. 57 (2006) 654.

[5] E. Epelbaum, H. W. Hammer and U.-G. Meißner, arXiv:0811.1338 [nucl-th].

[6] N. Fettes, U.-G. Meißner and S. Steininger, Nucl. Phys. A 640, 199 (1998).

[7] D. R. Entem and R. Machleidt, Phys. Rev. C 68, 041001 (2003).

[8] E. Epelbaum, W. Glöckle and U.-G. Meißner, Nucl. Phys. A 747, 362 (2005).

[9] A. Nogga, R. G. E. Timmermans and U. van Kolck, Phys. Rev. C 72 (2005) 054006.

[10] E. Epelbaum and U.-G. Meißner, arXiv:nucl-th/0609037.

[11] M. C. Birse, Phys. Rev. C 76 (2007) 034002.

[12] E. Ruiz Arriola, A. Calle Cordon and M. Pavon Valderrama, [arXiv:0710.2770 [nucl-th]].

[13] U. van Kolck, Phys. Rev. C 49, 2932 (1994).

[14] E. Epelbaum et al., Phys. Rev. C 66, 064001 (2002).

[15] A. Nogga, P. Navratil, B. R. Barrett and J. P. Vary, Phys. Rev. C 73, 064002 (2006).

[16] P. Navratil et al., Phys. Rev. Lett. 99, 042501 (2007).

[17] H. Witala et al., Phys. Rev. C 73 (2006) 044004.

[18] J. Ley et al., Phys. Rev. C 73, 064001 (2006).

[19] P. Navratil et al., Phys. Rev. Lett. 99 (2007) 042501.

[20] H. Polinder, J. Haidenbauer and U.-G. Meißner, Nucl. Phys. A 779 (2006) 244.

[21] H. Polinder, J. Haidenbauer and U.-G. Meißner, Phys. Lett. B 653 (2007) 29.

[22] D. E. Gonzalez Trotter et al., Phys. Rev. Lett. 83, 3788 (1999).

[23] V. Huhn et al., Phys. Rev. Lett. 85, 1190 (2000).

[24] V. Lensky et al., Eur. Phys. J. A 33 (2007) 339.

[25] A. Gardestig and D. R. Phillips, Phys. Rev. C 73 (2006) 014002.

[26] N. Kaiser, S. Gerstendorfer and W. Weise, Nucl. Phys. A 637 (1998) 395.

[27] H. Krebs, E. Epelbaum and U.-G. Meißner, Eur. Phys. J. A 32 (2007) 127.

[28] E. Epelbaum, H. Krebs and U.-G. Meißner, Nucl. Phys. A 806 (2008) 65.

[29] E. Epelbaum, H. Krebs and U.-G. Meißner, Phys. Rev. C 77 (2008) 034006. 